\title{
Comprensión y producción oral y escrita en la enseñanza del español para los tres ciclos de la educación secundaria mexicana: entre lo deseable y lo posible'
}

\author{
Oral and Written Expression and Perception. The case of Spanish Language \\ Teaching in the Three Stages of Mexican Secondary Education: \\ Between what is Desirable and what is Possible
}

\author{
GILBERTO FREGOSO PERALTA \\ Universidad de Guadalajara \\ México \\ fepg@hotmail.com
}

\section{LUZ EUGENIA AGUILAR GONZÁLEZ \\ Universidad de Guadalajara México \\ aguilar.luzeugenia@gmail.com}

(Recibido 22-05-20I5; aceptado 26-IO-2OI5)

Resumen. Se reporta el análisis de tres libros vigentes para la enseñanza del idioma español en los cursos respectivos de la asignatura, dentro del nivel educativo secundario mexicano. Motivaron la pesquisa dos propósitos: explorar sus contenidos e implementación, así como estimar si tal proceso sentaba un precedente idóneo para el trayecto académico posterior, ello al constatar de manera cotidiana las limitaciones que en materia de dominio del lenguaje muestran nuestros alumnos de licenciatura y posgrado, tanto en la comprensión como en la producción oral y escrita.

El resultado plantea un dilema susceptible de alimentar una polémica oportuna, de un lado, la propuesta en cuanto a contenidos y didáctica es ambiciosa, compleja y requiere el acceso a recursos lo mismo simbólicos que tecnológicos sofisticados, actuales y probablemente eficaces en ámbitos donde se disponga de ellos, de otro, para muchos entornos de nuestra realidad socioeconómica nacional sería excesiva, incumplible, descontextualizada.

Palabras clave: Libros; Enseñanza; Español; Secundaria; Dilema.
Abstract. The study presents an analysis of three books for teaching Spanish language in the Mexican Junior High Educational courses (Secondary level). This research sets two goals: to explore their content and application and to estimate whether this process establishes a suitable precedent for a later academic path. This is based upon the severe limitations observed in reading, writing and speaking in undergraduate and graduate university students. The result poses a dilemma capable of feeding a timely debate, on the one hand, the proposal in terms of content and teaching is ambitious, complex and requires access to sophisticated modern technology and symbolic resources. On the other hand, though it can be effective in areas where it is available, in others and because of the economic reality, it would be excessive, unenforceable and out of context.

Keywords: Junior High; Spanish; Teaching; Books.

\footnotetext{
I Para citar este artículo: : Fregoso Peralta, Gilberto y Aguilar González, Luz Eugenia (2OI5). Comprensión y producción oral y escrita en la enseñanza del español para los tres ciclos de la educación secundaria mexicana: entre lo deseable y lo posible. Alabe 12. [www.revistaalabe.com]

DOI: IO.I5645/Alabe.20I5.I2.6
} 


\section{I - Introducción}

El conjunto de los libros a reportar responde al plan y programas de español elaborados por la Secretaría de Educación Pública (SEP), pero editado por una empresa privada como ahora se estila. Para el ciclo 20I4 esta entidad federal había autorizado 25 títulos de español para cada uno de los tres cursos, publicados por i8 editoriales. Ante una oferta tan variada, se optó por escoger una edición para su estudio mediante sorteo, habiendo correspondido a Ediciones SM. Conecta palabras I, 2 y 3 Español Secundaria, en la inteligencia de no diferir sustancialmente unas versiones de otras por cuanto responden a un criterio curricular establecido por la SEP, tocante a propósitos, procedimientos y competencias a desarrollar. De los textos hemos procedido a registrar y analizar los bloques que como unidades mayores los constituyen; los proyectos de cada bloque, con sus respectivos marcos conceptuales y metodológicos, materiales de consulta, productos a generar y los criterios de evaluación (Castillo y otros, 2OI2).

\section{II - Elementos de contexto.}

El Instituto Nacional para la Evaluación de la Educación (INEE, 2OII) daba a conocer que al iniciarse la segunda década del siglo XXI, I3 millones $33^{2}$ mil infantes en nuestro país vivían en hogares donde el jefe de familia carecía de instrucción o sólo contaba con la primaria o secundaria incompleta. Que tal condición, fincada en la desigualdad y la pobreza, había evitado que terminaran la primaria $5^{\text {I }}$ por ciento de los adolescentes entre I5 y I7 años con un tutor sin escolaridad; 43.2 por ciento de quienes viven en zonas rurales y 42.9 por ciento de los indígenas en ese rango de edad.

Así mismo señalaba que de cada Ioo niños de I2 a I4 años, 49 no asistían a la escuela entre quienes realizaban una jornada de trabajo, ${ }_{5}$ de cada Ioo no acudían a un plantel al vivir en pobreza alimentaria y I3 de cada Ioo no asistía a una escuela en las zonas indígenas y rurales. Añadía que el derecho a la educación entre los críos de tres a cinco años y los adolescentes de i2 a I 7 no se ejerce a cabalidad en México y que su cumplimiento estaba asociado con las condiciones étnicas, económicas y de ubicación de residencia de los menores.

Según el organismo, un millón 200 mil jóvenes entre I5 y I7 años no cursaban la educación media superior, por lo que en el corto plazo el sistema educativo debía prepararse para atenderlos, sobre todo ante el entonces reciente decreto para establecer la obligatoriedad de ese nivel. En la población rural sólo cinco adolescentes por cada Io asistían a la educación media superior.

Con base en los indicadores del ciclo escolar 20IO/20II, se decía que 59·3 por ciento de los niños en primaria - 7 millones 897 mil 907 alumnos- y 36.7 por ciento de los adolescentes de secundaria-un millón 974 mil 242 estudiantes- asistían a escuelas en las que no se disponía de al menos una computadora con Internet. El acceso a dicha tecno- 
logía en los centros comunitarios adonde acude población indígena y rural era de apenas o.7 por ciento y el equipo no estaba conectado a la red.

La mitad de las primarias y una cuarta parte de las secundarias no contaban con al menos una computadora para el trabajo académico. En la educación media superior, $5^{6}$ por ciento de los planteles no cumplían con el parámetro de la Organización de Estados Americanos para la Educación -adoptado por México- de tener ocho estudiantes por computadora.

Los indicadores del sistema educativo evidenciaban la desigualdad enorme prevaleciente entre los mexicanos, así, apenas I5 por ciento de las telesecundarias con computadoras reportó tener conexión a Internet, en contraste con 86 por ciento de las secundarias generales. Entre entidades, federativas las brechas alcanzaban hasta 70 puntos. Por ejemplo, en el Distrito Federal, 9I.9 por ciento de las primarias disponían de este equipamiento, mientras en Zacatecas la cifra sólo llegaba a 20 por ciento. Al finalizar el ciclo 2OIO, 85.7 por ciento de los niños de I2 a I 4 años reportó tener la primaria completa; entre I5 y I7 años, la cifra fue menor (7o por ciento) respecto de la conclusión de la secundaria (INEE, 2OII).

Con datos de la SEP, la periodista Nurit Martínez, sostiene que la Red Nacional de Bibliotecas Públicas "está colapsada”, sobre todo debido a sus notorias deficiencias en materia de actualización de los acervos, mobiliario deteriorado, iluminación defectuosa y mínima capacitación del personal, situación que las ha convertido "en bodegas y no en centros de lectura”, con un promedio de tan sólo 25 visitas por día, para las diez mil públicas en todo el país. Por su parte, se contabilizaban 5 mil 3 OI escolares claramente improvisadas. (El Universal, o8/o9/2005).

En síntesis apretada, valga formarse una idea sobre el dominio del español académico observado entre el alumnado de la secundaria mexicana, acorde con el Cuadro i:

Tabla I: Resultados de las pruebas Enlace 2006 a $20 I$ de español, aplicadas a los alumnos de secundaria del país

\begin{tabular}{|l|l|l|l|l|}
\hline Año & Insuficiente & Elemental & Bueno & Excelente \\
\hline 2006 & $40.7 \%$ & $44.6 \%$ & $14.0 \%$ & $0.7 \%$ \\
\hline 2007 & $36.3 \%$ & $44.8 \%$ & $17.9 \%$ & $1.0 \%$ \\
\hline 2008 & $32.9 \%$ & $49.2 \%$ & $17.1 \%$ & $0.8 \%$ \\
\hline 2009 & $32.1 \%$ & $49.7 \%$ & $17.4 \%$ & $0.8 \%$ \\
\hline 2010 & $37.4 \%$ & $44.6 \%$ & $17.2 \%$ & $0.7 \%$ \\
\hline 2011 & $40.1 \%$ & $42.8 \%$ & $16.1 \%$ & $1.0 \%$ \\
\hline
\end{tabular}

Fuente: Evaluación Nacional del Logro Académico en Centros Escolares, Enlace $20 \mathrm{OII}$. www.enlace.sep.gob.mx/content/ba/pages/ jalisco icas.html 
El redondeo de los guarismos nos hace saber que ocho de cada diez educandos del nivel se ubican entre la insuficiencia y lo elemental. Con tales antecedentes, revisemos la propuesta de enseñanza del idioma nacional a partir de 2012.

\section{III - La propuesta de enseñanza en el libro para el ciclo I ${ }^{\circ}$ de la educación secundaria.}

La edición consta de 272 páginas, el segmento medular contiene cinco bloques teórico-prácticos integrados por proyectos (pp.I4-248), cuatro anexos (pp. 252-259) e igual número de actividades permanentes recomendadas para implementarse durante el curso (260-266); finalmente, la bibliografía consultada para todo el libro (268-272).

Inicia el primer bloque con el proyecto titulado Un buen comienzo...El reglamento de nuestro grupo que se propone en doce sesiones comprender la función regulatoria de tales documentos en las sociedades, analizar el contenido de reglamentos, así como emplear los modos y tiempos verbales apropiados para indicar derechos y responsabilidades al escribir textos normativos. El producto concreto a generar por el grupo organizado en equipos (sin precisar el número de integrantes) es un reglamento para el uso del salón.

El segundo proyecto se denomina De héroes, dioses y cosas sobrenaturales cuyo cometido es satisfacer en doce sesiones identificar las características de mitos y leyendas para establecer semejanzas y diferencias entre ambos tipos de textos, reconocer la función de mitos y leyendas con relación a los valores de un grupo social, comprender la importancia de la tradición oral como medio para conocer culturas diversas, distinguir las diferencias entre distintas versiones de un mismo mito o leyenda en función del grupo social al que pertenece.

En equipos, sin especificar la cantidad de miembros, los estudiantes deberán compilar muestras de ambos tipos de relatos, escritos o producto de entrevistas orales, para difundirlos editados en versión impresa o audiovisual (libro impreso o manuscrito, grabación de audio o vídeo, producto multimedia).

El último proyecto del primer bloque se llama Escribir para sistematizar información y explicita la expectativa, en doce sesiones, de analizar deferentes materiales de consulta a fin de obtener información considerando la organización del texto y sus componentes, elaborar fichas de trabajo utilizando paráfrasis y recursos gráficos, escribirlas de acuerdo con propósitos específicos y citar las fuentes consultadas, emplear el resumen como un medio para seleccionar, recuperar y organizar información. Se pide trabajar en equipos de tres alumnos para seleccionar un tema, localizar y reunir datos sobre él mediante fichas de trabajo.

La evaluación del Bloque I, anunciada como TIPO PISA (Programa Internacional de Evaluación Estudiantil, por sus siglas en inglés) propone la lectura de cuatro textos y se formulan diez preguntas de opción múltiple. Exploremos el bloque II.

El cuarto proyecto del libro, primero del segundo bloque, se titula Comunicar lo que se sabe, cuya misión es lograr en doce sesiones los aprendizajes interpretar la información contenida en fuentes de consulta y emplearlas al redactar un texto, recuperar las 
características textuales de monografías, utilizar nexos que organizan, ponderan e introducen ideas en un texto, emplear la tercera persona, el impersonal y la voz pasiva en la descripción de los fenómenos. Se trata ahora de redactar una monografía producto de una investigación sobre un tema de interés, con apoyo en las fichas elaboradas durante el proyecto tercero e integrados los educandos en el mismo equipo de tres miembros, la expectativa es que sus pares de generaciones posteriores consulten dicho trabajo.

Nótese el nivel de la exigencia para adolescentes mexicanos de educación media básica, tan vapuleados en las pruebas PISA y ENLACE aplicadas por la Organización para la Cooperación y el Desarrollo Económicos (OCDE) y la Secretaría de Educación Pública, respectivamente (Fregoso y Aguilar, 20I4).

El quinto proyecto, segundo del bloque II, se titula Cuéntame una historia... de ciencia ficción y formula los aprendizajes siguientes en ocho sesiones: analizar los recursos literarios y discursivos empleados en los cuentos de ciencia ficción e identificar el papel de ciencia y tecnología en dicho género. En equipos de tres estudiantes, leerán narraciones de este tipo, analizarán los recursos literarios y discursivos, detectarán la presencia de la ciencia y la tecnología en la trama, escribirán un cuento propio para socializarlo entre parientes y conocidos.

El proyecto Un suceso, varias posturas y un debate, el sexto en el libro y tercero del bloque II, espera cumplir estos aprendizajes en ocho sesiones: comparar el tratamiento de una misma noticia en distintos medios, recuperar los datos de las fuentes consultadas al analizar una noticia, argumentar los puntos de vista propios al analizar una noticia y expresar una opinión sobre los hechos referidos, utilizar tecnologías de la información y comunicación como fuentes. La actividad final consiste en revisar y comparar noticias, sustentar una opinión con argumentos en un debate organizado por el grupo. Se pide trabajar en equipos, pero sin determinar el número de sus integrantes.

Tal vez hubiera sido más sencillo detectar el tratamiento específico diferencial de cada medio a través de la consulta de géneros de opinión como el editorial, la columna, la caricatura, el artículo.

El aspecto central de la evaluación del bloque segundo, también anunciada TIPO $P I S A$, plantea a los chicos la lectura de cuatro textos, tras hacerlo se les formulan diez preguntas de opción múltiple. Veamos ahora el bloque III.

El séptimo proyecto del libro y primero del bloque tercero, Exponer lo investigado, se propone en seis sesiones seleccionar información de un tema para presentarla en una exposición oral, organizar la información para participar, emplear recursos discursivos y prosódicos orales para mantener la atención de la audiencia al exponer los resultados de una investigación, usar las TIC como recurso para exponer y como fuente de información. Se trata ahora de desarrollar una exposición oral fincada en la información producto de la pesquisa realizada durante los proyectos 3 y 4 , con los mismos compañeros de equipo.

El octavo proyecto, segundo del bloque III, Un laboratorio de poesía, espera, en catorce sesiones, conocer algunas características de la poesía de vanguardia del siglo XX, analizar el lenguaje figurado y el efecto de los recursos sonoros y gráficos en los poemas, 
emplear recursos literarios para plasmar estados de ánimo y sentimientos en la escritura de poemas. El quehacer productivo consistirá en escribir un poema personal inspirado en los movimientos de vanguardia del siglo pasado, para luego leerlo en voz alta.

El proyecto noveno Una carta formal-último del bloque tercero- tiene como propósito aprender en diez sesiones a identificar las características y función de las cartas formales, emplear éstas para aclaraciones, solicitudes o reclamos y recuperar información para sustentarlas. Se prescribe a cada quien redactar una carta tendiente a solucionar un problema de la comunidad donde se habita.

La evaluación del Bloque III, inspirada en la OCDE, pide a los chicos leer tres textos, para luego contestar trece reactivos cerrados. Pasemos al bloque IV.

El décimo proyecto, titulado Corregir para mejorar, pretende en ocho sesiones conocer las características y función de un informe de investigación, sistematizar información sobre un proceso estudiado, manejar los conectores para establecer relaciones temporales, aplicar recursos gramaticales para dar cohesión al texto. La actividad concreta -en equipos de tres alumnos- implica redactar un informe de investigación con las notas obtenidas durante alguna actividad realizada para la materia de biología.

Por su parte, el décimo primer proyecto, Del cielo cayó un pañuelo. Lírica tradicional mexicana, establece tres aprendizajes en doce sesiones: interpretar el significado de tales textos y emplear recursos prosódicos para leerlos en voz alta, identificar sus características, conocer y valorar la riqueza lingüística y cultural de México mediante ellos. El afán tangible del proyecto solicita un escrito individual de carácter lírico a ser compartido oralmente con los compañeros y presentado a la comunidad mediante un recital.

El proyecto doce, Mirar televisión... análisis de contenidos televisivos, espera tres aprendizajes en ocho sesiones: analizar los programas y argumentar una opinión personal, conocer criterios para el análisis de la información, evaluar su influencia en las personas. Esta vez se trata de elegir un programa para analizar su contenido y redactar una reseña crítica donde se argumente una opinión personal, la que habrá de difundirse en el periódico mural de la escuela o en una antología. Nuestros alumnos de la universidad no serían capaces de cumplir tal exigencia.

El segmento que evalúa el bloque cuarto, así mismo TIPO PISA, solicitó la lectura de cuatro escritos para responder catorce preguntas, sólo seis de ellas abiertas. Pasemos revista al último bloque del libro, el $\mathrm{V}$.

El décimo tercer proyecto, Lo nuestro es puro teatro, tiene como objetivo tres aprendizajes en doce sesiones: características de las obras de teatro, discriminar sus elementos para adaptarla, aplicaros los signos de puntuación y acotaciones para el libreto. Los estudiantes se enteran de que deberán leer varias obras dramáticas clásicas y, en equipos de tres, adaptar una de ellas a la época actual mediante un guion.

El último de los proyectos, Una influencia enriquecedora, plantea como aprendizajes en ocho sesiones identificar y valorar la variedad cultural y lingüística del país, comprender la influencia de las lenguas indígenas en el español actual, colegir su valor en la identidad nacional. Procede en esta ocasión indagar sobre la riqueza lingüística de 
la nación para valorar la influencia de las hablas indígenas en el español, ello para redactar un texto expositivo acerca de las palabras provenientes de los idiomas vernáculos y la caracterización de los pueblos que los hablan, escrito a difundirse en el periódico mural de la escuela. Se pide trabajar en equipos, pero sin sugerir de cuántos miembros cada uno.

La evaluación del bloque $\mathrm{V}$ requirió la lectura de cuatro escritos a fin de contestar I7 preguntas, sólo seis de ellas abiertas.

\section{Observaciones críticas.}

Acentuación, puntuación, conectores y cómo corregir los escritos son cuatro anexos que se aúnan a la reglamentación para llevar acabo las actividades permanentes sugeridas para el grupo: conferencias, club de lectura, cineclub, taller de teatro (deseables a no dudar, ¿pero factibles en términos de espacio y equipamiento?), y a la bibliografía para cada proyecto, de la que surge una duda más: ¿será conseguible en la mayor parte de las poblaciones del país?

De este primer libro pareciera poco probable que en muy diversos puntos de nuestra geografía -zonas urbanas y rurales marginadas- los chicos estuvieran en condiciones de imprimir o de grabar en audio o video el producto de su trabajo (proyecto 2); de redactar una monografía sobre un tema con la expectativa de legarla a las generaciones posteriores (proyecto 3); discernir el tratamiento específico conferido a una misma noticia en diferentes medios informativos (proyecto 6); elaborar una ponencia para exponerla oralmente mediante el uso de paráfrasis (proyecto 7); elaborar una reseña sobre un programa televisivo luego de analizarlo y entregar dicho producto para una antología generada por el grupo (proyecto I2), adaptar una obra dramática clásica a la época moderna, guion de por medio (proyecto I3).

Como profesores suscribiríamos con entusiasmo la expectativa de que el alumnado desarrollara el cúmulo de aptitudes y productos estipulados, pero aunado a la carencia de infraestructura, algunos de los trabajos pedidos con dificultad los podrían solventar jóvenes universitarios, no los adolescentes que cursan el primer año del nivel medio básico, de manera puntual quienes lo hacen en ambientes donde se carece de formación previa suficiente, biblioteca, equipamiento, capacitación y señal de internet, personal académico ad hoc a la asignatura impartida, librerías, progenitores con escolaridad y tiempo para apoyar las tareas.

\section{IV - La propuesta de enseñanza en el libro para el ciclo $2{ }^{\circ}$ de la educación secundaria.}

La edición consta de 272 páginas, el segmento medular contiene cinco bloques teóricoprácticos integrados por proyectos (pp.I4-257), tres recomendaciones para actividades permanentes a ser implementadas durante el curso (pp. 258-263), igual número de anexos con información para consulta (pp. 264-270), finalmente, la bibliografía para todo el libro (271-272). 
Inicia el primer bloque con el proyecto titulado Escritura breve, efectos grandes: escribir artículos que espera desarrollar en doce sesiones la capacidad en los chicos de contrastar las distintas formas de tratar un mismo tema en diferentes fuentes, integrar la información de distintas fuentes para producir un texto propio, emplear explicaciones, paráfrasis, ejemplos, repeticiones y citas para desarrollar ideas en un texto. Tras leer varios artículos y haber buscado información pertinente, el producto concreto a generar es un texto expositivo o argumentativo del género artículo y darlo a conocer en una revista (¡) del grupo. Ello, organizados en triadas.

El segundo proyecto se denomina Leer para comentar y su cometido es satisfacer en doce sesiones los aprendizajes analizar el ambiente y rasgos de los personajes de cuentos latinoamericanos, identificar las variantes sociales, culturales o dialectales en los textos conforme la época y lugares descritos, identificar los recursos empleados para describir aspectos espaciales y temporales que crean el ambiente en un cuento y elaborar comentarios de un cuento a partir de su análisis e interpretación. Así, tras buscar y leer relatos cortos de autores de nuestro subcontinente, cada educando escogerá uno para analizarlo, redactar un comentario y leerlo ante el grupo.

El último proyecto del primer bloque se llama Investigo, conozco y defiendo mis derechos y explicita la expectativa de tres aprendizajes en doce sesiones: interpretar documentos sobre derechos humanos y reconocer su importancia en la regulación de las sociedades, identificar los documentos nacionales e internacionales sobre el tema, identificar los modos y tiempos verbales que se utilizan en los documentos nacionales e internacionales sobre el particular. Se pide consultar y acopiar información sobre el asunto para diseñar un cartel en equipo de tres compañeros, a fin de exhibirlo en una jornada escolar dedicada al tema.

El núcleo de la evaluación del Bloque I, anunciada como TIPO PISA, propone la lectura de dos escritos y se formulan once preguntas, nueve abiertas y dos de opción múltiple. Pasemos al bloque II.

El cuarto proyecto (primero del segundo bloque) se titula Una discusión formal: la mesa redonda, cuya expectativa es lograr en doce sesiones que los jovencitos revisen y seleccionen información de textos diversos para participar en una mesa redonda, argumenten sus puntos de vista y utilicen recursos discursivos al intervenir en discusiones formales para defender sus opiniones, recuperen información y puntos de vista que aporten a la discusión, así como llegar a conclusiones sobre un tema. Procede ahora recopilar información sobre algún asunto para formarse una opinión y darla a conocer durante una mesa redonda organizada por el grupo. Los equipos que participarán estarán formados por cinco chicos.

El quinto proyecto, segundo del bloque II, se titula Cuentos y recuentos y formula tres aprendizajes en diez sesiones: a) Modificar las características de los diferentes aspectos, en función de la historia que presenta un cuento original. b) Modificar la estructura del cuento e identificar sus implicaciones en el efecto que causa. c) Emplear recursos lingüísticos para describir personajes, escenarios y situaciones. Cada tres alumnos es- 
cogerán un cuento al que le harán modificaciones, para luego compartirlo con los demás compañeros en plenaria grupal.

El proyecto ;Guagua, bus! Hablamos de lo mismo, pero diferente... el sexto en el libro y tercero del bloque II, contempla cumplir tres objetivos en ocho sesiones: identificar que una misma expresión o palabra puede tener distintos significados en función del contexto social y geográfico, reconocer la importancia del respeto a la diversidad lingüística e identificar la variedad léxica de los pueblos hispanoparlantes como parte de la riqueza lingüística y cultural de nuestro idioma. Esta vez el producto consiste en elaborar una tabla comparativa con vocablos distintos, de lugares diversos, para nombrar un mismo referente, como empleo del español en culturas múltiples. Los equipos integrarán a entre cuatro y cinco educandos.

El aspecto central de la evaluación del bloque segundo, también anunciada TIPO $P I S A$, plantea a los chicos la lectura de un texto, tras hacerlo se le formulan cinco preguntas: tres abiertas y dos de opción múltiple. Pasemos al bloque III.

El séptimo proyecto del libro y primero del bloque tercero, Un ensayo literario, propone como aprendizaje en diez sesiones identificar semejanzas y diferencias en la manera de tratar un mismo tema en distintos textos literarios y autores, emplear el ensayo como medio para plasmar su análisis y posicionar su punto de vista, identificar el uso del lenguaje en el tratamiento de un tema en diferentes géneros. Se trata de elegir y consultar escritos sobre un mismo tema para elaborar un ensayo literario personal con la opinión de cada alumno, publicar algunos en la revista del grupo $(j !)$ y armar una antología.

El octavo proyecto, Un personaje, una vida, un texto, segundo del bloque III, tiene la expectativa de cuatro aprendizajes en doce sesiones: seleccionar datos y sucesos más importantes de la vida de un personaje, utilizar adecuadamente recursos lingüísticos, modos y tiempos verbales en la redacción de biografías, emplear sinónimos y pronombres para referirse a los objetos que se mencionan reiteradamente, emplear adjetivos, participios y aposiciones en la descripción de los personajes. Se requiere del alumnado escribir, cada bina, la biografía de algún literato.

El proyecto noveno, Dibujar para cuestionar y opinar, es el último del tercer bloque y tiene como propósito aprender en doce sesiones a identificar la caricatura como una forma de presentar una noticia en la prensa escrita, reconocer la influencia de la caricatura y el efecto que causa en la sociedad, adoptar una postura crítica sobre la forma en que se presentan las noticias en la caricatura periodística. Aquí se prescribe consultar información periodística pertinente a modo de valorar la función de la caricatura de opinión y formarse un criterio para dibujar personalmente una, incluirla en la revista del grupo y montar con todas una exposición. El dibujo se pretende individual y el montaje colectivo.

La evaluación del Bloque III se anuncia como TIPO PISA e implica leer un texto tras lo cual se deben contestar nueve preguntas, cuatro de opción múltiple y cinco abiertas. Vayamos al Bloque IV.

El décimo proyecto, titulado Dialogar para construir conocimientos, pretende en 
ocho sesiones lograr que el estudiante identifique las características de las entrevistas y su función como fuente de información, discrimine la información que debe incluir un reporte de entrevista y sea capaz de emplear el diálogo directo y la narración al redactarlo, respete la integridad del entrevistado durante la interlocución y al elaborar el informe de esta. Entrevistar a un especialista o persona enterada sobre algún tema interesante para redactar un reporte y difundirlo en el periódico mural o vía internet, es la actividad prescrita en el proyecto. Tres alumnos por equipo.

Llegamos al décimo primer proyecto, Te recomiendo... una novela, que espera en doce sesiones los aprendizajes interpretar el significado de una novela, identificar la función de las reseñas literarias como recurso para difundir una obra, utilizar recursos discursivos para generar el interés del lector, emplear algunos aspectos clave de la historia y datos sobresalientes del autor al redactar una reseña. La producción concreta señala, para equipos de tres estudiantes, elegir una novela y redactar la reseña respectiva, para después compilar todas las del grupo en una antología y proceder a leerlas en voz alta en plenaria, así como editar otras en las revistas del grupo. Nada menos.

El proyecto doce, Investigar, escribir, reportar tiene la expectativa de tres aprendizajes en doce sesiones: precisar los propósitos y el punto de vista del autor en reportajes leídos, identificar las características y función de los reportajes, integrar información de diversas fuentes al escribir un reportaje, a más de atender a las características del tipo de texto. La expresión tangible entraña organizar equipos de tres integrantes para elaborar un reportaje sobre un tema de interés público para difundirlo en alguna de las revistas del grupo (¡!) o en el periódico mural de la escuela.

El apartado que evalúa el bloque cuarto, así mismo TIPO PISA, solicitó la lectura de tres escritos para responder trece preguntas, apenas dos de ellas de opción. Pasemos revista al último bloque del libro para el segundo nivel de la secundaria, el V.

El décimo tercer proyecto, Así ocurrió, así pasó contempla en diez sesiones los aprendizajes conocer las características y función de la crónica y aplicarlas al narrar un suceso, emplear referencias de tiempo, espacio y persona al redactar una crónica, emplear recursos lingüísticos y discursivos al redactarla. Reunidos en triadas, los jovencitos deberán leer varios textos de este género para luego redactar uno, a publicarse en alguna revista del grupo, en el periódico mural o en Internet.

El último de los proyectos, Papelito habla, pretende en ocho sesiones interpretar el contenido de la carta poder y reconocer su carácter legal, redactar una en los términos legales y temporales que preserven su seguridad, analizar los términos legales en ella y las consecuencias que se derivan de estos, valorar la importancia de contar con una firma estable como recurso para acreditar la identidad personal. El producto tangible se elaborará en binas y consistirá en redactar una carta poder tras entender su función. La evaluación del bloque V (proyectos I3 y I4) requirió la lectura de dos escritos a fin de contestar Io preguntas, la mitad de ellas abiertas.

Esta vez se propusieron tres actividades permanentes: Instaurar un Club de Lectores Críticos con el objetivo de editar una revista por parte del grupo, establecer un Taller 
de Escritura para fomentar las actividades de redacción, así como generar un taller de edición como apoyo técnico a la elaboración de la pretendida revista. Sugerencias de lo más atractivas, ¿pero factibles en términos de espacio y equipamiento? Tocante a los anexos, el primero aborda el tema de la argumentación en el discurso con referencia al debate; el segundo centra el interés en el discurso de carácter expositivo o explicativo. En ambos se hace énfasis en su estructura y función respectiva, así como en los conectores que les son más afines.

\section{Consideraciones críticas}

Nuevamente el conjunto de actividades como propuesta es loable pero poco creíble su realización, por ejemplo, la de conseguir la bibliografía consignada para cada proyecto e ir más allá de la información contenida en el tomo 2, ¿estará disponible en la mayor parte de las poblaciones del país?

Del segundo libro pareciera poco probable que en muy diversos puntos de nuestra geografía -zonas urbanas y rurales marginadas- los chicos tuvieran la aptitud de elaborar un artículo expositivo o argumentativo y después publicarlo en una revista del grupo, planteamiento por demás irreal (proyecto I), crear un ensayo literario para su edición en una revista del grupo y luego en una compilación, nada menos (proyecto 7), diseñar la biografía de un literato famoso, lo que pareciera más una invitación a transcribirla (proyecto 8), dibujar una caricatura de opinión y publicarla -por supuesto- en alguna de las pretendidas revista grupal (proyecto 9), entrevistar a un especialista en algún tema de actualidad y difundirla en el periódico mural de la escuela y por internet (proyecto Io), hacer la reseña crítica de una novela, publicarla ya sabemos dónde y reunirla en una antología del grupo (proyecto II), escribir un reportaje sobre algún tema de interés, complementado con el material fotográfico respectivo y difundirlo a través del periódico mural y la consabida revista (proyecto I2), ídem con una crónica (proyecto I3).

¿Qué mentor no estaría de acuerdo en volver realidad tal ensoñación? No estaría por demás explorar en la teoría sobre la conciencia posible formulada por Lucien Goldmann (I975), a modo de estimar los montos de cambio cultural exigibles a los sujetos y no fracasar en el intento.

\section{V-La propuesta de enseñanza en el libro para el ciclo $3{ }^{\circ}$ de la educación secundaria.}

La edición consta de 263 páginas, el segmento medular contiene cinco bloques teórico-prácticos integrados por proyectos (pp.I6-239), tres recomendaciones para actividades permanentes a ser implementadas durante el periodo (pp. 253-258), cinco anexos con información para consulta (pp. 244-252), finalmente, la bibliografía del libro (259-263). 
Inicia el primer bloque con el proyecto titulado Comunicar puntos de vista que pretende desarrollar en diez sesiones los aprendizajes conocer las características y la función de los ensayos, contrastar la información obtenida en distintos textos e integrarla para complementarla, reconocer el punto de vista del autor y diferenciar entre datos, opiniones y argumentos en un texto, argumentar los puntos de vista personales respecto al tema que el alumno desarrolla en un ensayo y sustenta con información de las fuentes consultadas. Los chicos, organizados en binas, deben leer documentos con opiniones diversas sobre una misma situación a modo de fundamentar sus argumentos propios, redactar un ensayo, compilar los de todo el grupo para que otras personas los lean, difundir algunos por internet o en el periódico mural.

El segundo proyecto se denomina Poesía y movimientos literarios, su cometido es satisfacer en diez sesiones las expectativas reconocer el contexto histórico y social de la poesía dentro de un movimiento literario, identificar la función y las características de las figuras retóricas en los poemas a partir de un movimiento literario, analizar el lenguaje figurado en los poemas. Tras conocer los más importantes movimientos poéticos generados a lo largo de la historia, los alumnos en equipos de tres, buscarán ejemplos de poemas a fin de identificar su temática y su estructura para ubicarlos en el movimiento literario respectivo. Ello, con el propósito de exponer oralmente ante el grupo ese análisis y leer fragmentos de poemas representativos.

El último proyecto del primer bloque se titula Una lectura crítica de los anuncios publicitarios y explicita lograr tres aprendizajes en diez sesiones: analizar las características de los mensajes publicitarios, identificar el efecto de los mensajes publicitarios en los consumidores, precisar las características y funciones de los recursos lingüísticos y visuales que se utilizan en los anuncios publicitarios, describir el impacto de los anuncios publicitarios en la sociedad a través de un texto, también revisar, interpretar y organizar los resultados de una encuesta, mediante un informe. En equipos de tres jovencitos, se pide valorar críticamente (¡!) los mensajes de este tipo y aplicar encuestas para estimar la influencia de la publicidad en los gustos y formas del consumo social.

La evaluación del Bloque I, anunciada como TIPO PISA, propone la lectura de dos escritos, uno argumentativo y otro poético, para luego contestar trece preguntas, de las que nueve son de opción múltiple. Pasemos al bloque II.

El cuarto proyecto (primero del segundo bloque) se titula Participar en un panel, cuya expectativa es lograr en ocho sesiones identificar la diferencia entre los argumentos basados en datos y los basados en opiniones personales, expresar de manera clara los argumentos y sustentarlos en información analizada, al debatir sobre un tema, utilizar recursos discursivos para persuadir y defender su posición en un panel de discusión, reconocer y respetar los diferentes puntos de vista sobre un tema, así como respetar los turnos de participación al llevar a cabo un panel de discusión. Procede esta vez comparecer en el susodicho para exponer un tema analizado y dar a conocer opiniones argumentadas, lo anterior mediante equipos de cuatro integrantes.

El quinto proyecto, segundo del bloque II, se titula Leer, escribir y compartir: 
antologías y prólogos para lo cual formula tres aprendizajes en diez sesiones: analizar e identificar la información presentada en textos introductorios (prólogos, reseñas, dedicatorias, presentaciones, utilizar la información de un prólogo para anticipar el contenido, los propósitos, y las características de una obra literaria o una antología, determinar el lenguaje adecuado (directo o indirecto) para dirigirse a los lectores al redactar un prólogo. Ahora, organizados en equipos de tres educandos, elaborarán una antología con textos literarios cortos y la prologarán.

El proyecto Solicitudes y usos de formularios es el sexto en el libro y tercero del bloque II, contempla cumplir en diez sesiones los aprendizajes: comprender los requisitos de información y documentación que requiere el llenado de un formulario y los documentos probatorios adicionales que se solicitan, emplear información contenida en documentos oficiales para el llenado de formularios, verificar que la información que reporta sea completa y pertinente con lo solicitado, reconocer la utilidad de los medios electrónicos para la realización de trámites. En equipos de tres, los estudiantes redactarán un reporte escrito tras averiguar qué trámites y cuáles documentos se necesitan para: gestionar el ingreso al bachillerato, llenar una solicitud de trabajo, obtener el pasaporte, el ingreso a una institución deportiva o cultural,

El aspecto central de la evaluación del bloque segundo, también anunciada TIPO $P I S A$, plantea a los educandos la lectura de un texto explicativo y contestar diez reactivos: seis de opción múltiple y cuatro abiertos. Veamos ahora el bloque III.

El séptimo proyecto del libro e inicial del bloque tercero, Informes de experimentos, espera en ocho sesiones elaborar el reporte de experimentos científicos utilizando adecuadamente el vocabulario técnico, los tiempos verbales y la concordancia sintáctica, describir los procesos observados y presentarlos de manera organizada, emplear recursos gráficos para presentar datos y resultados en un informe. Se trata de escribir el informe de un experimento realizado en la clase de ciencias, para lo que será necesario valerse de las notas tomadas durante tal curso. El trabajo es en binas.

Una mirada al pasado...lectura de textos renacentistas es el octavo proyecto, segundo del bloque III, y estipula cuatro aprendizajes en diez sesiones: inferir algunas características del Renacimiento a partir del análisis de una obra literaria, establecer relaciones entre las acciones de los personajes y las circunstancias sociales de la época, identificar la forma en que la literatura refleja el contexto social en que se produce la obra, emplear las TIC como fuente de información. Esta vez organizados en equipo de tres integrantes, la producción tangible implica leer y analizar obras literarias del Renacimiento español, para luego redactar un texto donde se describan aspectos del ámbito social reflejados en la obra literaria.

El proyecto noveno, Un programa de radio, es el último del tercer bloque y tiene como propósito aprender en doce sesiones a identificar y realizar los cambios necesarios para transitar del lenguaje escrito al oral empleando recursos prosódicos, jerarquizar y discriminar información para producir un programa radiofónico, valorar la utilidad de las TIC como fuentes y medio de información al producir un programa de radio, valorar 
y respetar las diversas manifestaciones culturales del mundo. Equipos de seis alumnos indagarán sobre una cultura particular a fin de diseñar un texto radiofónico $(j !)$ que verse sobre ella, adaptando el discurso a este medio.

La evaluación del Bloque III se anuncia como TIPO PISA e implica leer un guion radiofónico para responder cinco reactivos de opción. Acto seguido leer un escrito expositivo y contestar cuatro preguntas de opción y una abierta. Vayamos al Bloque IV.

Construir definiciones: mapas y crucigramas es el décimo proyecto y se propone en ocho sesiones aprender a abstraer información de un texto para elaborar definiciones de conceptos, utilizar el concepto de sinónimo y antónimo como recurso para construir crucigramas, establecer relaciones entre ideas en un mapa conceptual. El producto, en binas, es un mapa conceptual sobre un tema y además diseñar un crucigrama con las palabras contenidas en el mapa, material a compartir en plenaria de grupo.

Por su parte, el décimo primer proyecto, Una lectura dramatizada, establece dos aprendizajes en ocho sesiones: reconocer algunos valores de la época en que fue escrita la obra leída, comprender la importancia de la entonación y dramatización para darle sentido al lenguaje escrito en las obras dramáticas. El quehacer concreto comprende la lectura de una obra teatral en equipos de ocho a diez chicos, para después realizar una lectura actuada de varios pasajes valiéndose de la voz y la expresión corporal.

El proyecto doce, Un problema social y una historieta, plantea la exigencia de cuatro aprendizajes en doce sesiones: reconocer la importancia de recabar información sobre los antecedentes de un problema para resolverlo, emplear las onomatopeyas y aliteraciones para la exaltación de los significados, aplicar recursos gráficos y visuales para la construcción de un escrito, elaborar distintos textos para difundir información. La porción productiva entraña la lectura de historietas como ejemplos para elaborar una de manera individual, la que deberá figurar en una exposición organizada por el grupo y luego difundida en varios medios.

El apartado que evalúa el bloque cuarto, así mismo TIPO PISA, solicitó la lectura de dos escritos, uno expositivo/argumentativo y otro historietístico, para contestar siete reactivos de opción múltiple y cinco abiertos. Pasemos revista al último bloque del libro, el V.

Cuéntame cómo te ha ido... es el décimo tercer proyecto y primero de este nuevo bloque, estipula tres aprendizajes en ocho sesiones: sistematizar los pasajes más relevantes en la vida de cada alumno para elaborar una autobiografía, identificar las repeticiones excesivas y así evitarlas mediante la sustitución léxica y pronominal, jerarquizar las acciones de la autobiografía en un orden cronológico y coherente. Se trata de que cada estudiante escriba su propia historia personal para integrarla luego en anuario del grupo.

El último de los proyectos contenidos en el libro de texto para $3^{\circ}$ de secundaria se titula Para opinar y persuadir: un artículos de opinión, es el décimo cuarto y segundo del bloque $\mathrm{V}$, pretende en diez sesiones comprender el propósito comunicativo, el argumento y la postura del autor al leer artículos de opinión, escribir artículos de opinión argumentando su punto de vista y asumiendo una postura clara con el tema, recuperar información de diversas fuentes para apoyar sus argumentos y puntos de vista, jerarquizar 
información para expresar opiniones personales y contrastar ideas. Los alumnos leerán escritos de este género y cada quien redactará el propio para publicarlo en un periódico o revista escolar.

La evaluación del bloque V, TIPO PISA, requirió la lectura de dos artículos de opinión a fin de contestar Io preguntas, siete de ellas abiertas y tres de opción múltiple.

Como colofón, se sugieren tres actividades permanentes: Instaurar un Taller de lectura y producción de textos argumentativos con el propósito de estructurar el pensamiento propio y comprender el ajeno. Establecer un Club de lectura de textos informativos, con la expectativa de prohijar la competencia lectora de talante técnico y científico. Implementar un Club de lectura de textos literarios, a modo de enriquecer el gusto por la narrativa y compartir la experiencia con otras personas.

Tocante a los anexos, el primero aborda el tema de los textos argumentativos; el segundo centra la atención en las figuras retóricas; el tercero expone el uso de signos para separar e incorporar ideas en los párrafos; el penúltimo, cuarto, teoriza los aspectos de cohesión y coherencia; el quinto anexo hace un repaso de los signos de puntuación.

\section{Elementos de crítica}

El conjunto de actividades propuestas son a no dudar deseables, donde surge la duda es en su factibilidad y en la de conseguir la bibliografía consignada para cada proyecto, como decíamos respecto al volumen $2^{\circ}$, ¿será conseguible en la mayor parte de las poblaciones del país? Así concluye la propuesta editorial para el tercer curso.

Del tercer texto consideramos poco factible, y no deja de ser una opinión entre otras muy diversas, que la gran mayoría de educandos del nivel secundario esté capacitado para redactar un ensayo propio, difundirlo impreso y unirlo a otros en una antología (proyecto I), buscar ejemplos de poemas específicos de numerosas corrientes, analizarlos y exponer de manera oral sus conclusiones aderezadas con ejemplos (proyecto 2), valoración crítica de los mensajes publicitarios para estimar su influencia en las audiencias, aplicar encuesta al respecto y difundir resultados (proyecto 4), analizar obras literarias del Renacimiento español e inferir de ellas peculiaridades del ámbito en que fueron escritas (proyecto 8), indagación sobre una cultura particular para el diseño de material radiofónico que verse sobre ella, adoptando el discurso de este medio (proyecto 9), construcción de un mapa conceptual y de un crucigrama sobre un tema (proyecto Io), diseño de una historieta (trama, diálogos, dibujos) para difundirla en varios medios (I2), artículo de opinión a ser publicado en un periódico o una revista escolar (proyecto I4). 


\section{VI - Conclusiones}

Aunado a las observaciones y argumentación crítica externada al final de cada libro revisado, cerramos el análisis con varias consideraciones atinentes al conjunto.

Sin duda, las variadas prácticas discursivas y genéricas escritas, orales y de lectura solicitadas a los educandos son instrumento para su desarrollo intelectual y de indiscutible valor para su decurso académico posterior, por supuesto, en caso de haberlas desarrollado. Empero, nos pareció abrumadora la exigencia -cuantitativa y cualitativa- planteada en los libros abordados. La mayor parte de las prescripciones son notoriamente dirigidas a estudiantes de ambientes urbanos y de estratos con disponibilidad de recursos simbólicos y tecnológicos complejos.

Se sugiere a los chicos buscar información sobre temas diversos en la "biblioteca del salón”, ¿la habrá en el grueso de las escuelas? Nos surge la duda de si es posible cumplir con la variedad de las actividades reseñadas y productos solicitados, al considerar que simultáneamente a la de español cursan otras asignaturas. Llama la atención que el procedimiento evaluativo no se centre en las habilidades de producción oral y escrita promovidas, sino en la lectura de comprensión y con reactivos de opción múltiple. En algunos pasajes pareciera confundirse la mera búsqueda y consulta de información con la producción de conocimiento nuevo o, en sentido estricto, investigación. Por cierto, el nivel de exigencia pareciera implicar más tiempo del sugerido para algunas actividades de mayor esfuerzo intelectual (monografía, informe de investigación, adaptación teatral, elaboración de artículos, ensayos, encuestas).

Ignoramos qué significado pueda revestir hablar de la revista o revistas del grupo como el canal preciso para publicar las producciones escritas solicitadas a los chicos en varios de los proyectos, pues de sobra sabemos la responsabilidad implicada en una edición de este tipo, más si se le considera permanente.

Sin demérito del material revisado, cabe preguntarse si el estudiante típico mexicano de nivel medio básico está en aptitud de satisfacer lo que se le solicita o si las prescripciones de los autores pecan por exceso. Nuestra experiencia en la educación superior de licenciatura y posgrado nos impele a considerar como poco realista la expectativa de los libros estudiados, merced a las limitaciones referidas al capital cultural en términos de acceso a la información especializada y de dominio del lenguaje por parte de los alumnos universitarios, cuantimás de secundaria. Conviene al respecto revisar algunos estudios publicados recientemente, en los que resalta la similitud de falencias entre los chicos de la secundaria y los de las licenciaturas dentro del sistema educativo mexicano. Tan dramático como eso. (Arriaga, 2OI5; Fregoso y Aguilar, 20I4; Munguía Irma y otros, 2OI5). 


\section{Fuentes de consulta}

- Arriaga, R. (20Iо, febrero). Involución de la complejidad lingüística de los estudiantes mexicanos. De lingüis, Revista digital de idiomas, 4. Obtenido el I8 de agosto de $20 \mathrm{O} 2$ desde http://www.delinguis.unam.mx

- Arriaga, R. (20I5). Comprensión y redacción de textos de economía. México: UNAM.

- Castillo, A. y otros. (20I2). Español 1, 2, 3. México: conect@ palabras.

- Fregoso, G. \& Aguilar, L. E. (2OI4). La enseñanza del español en los textos escolares para el nivel primaria. Guadalajara: Ediciones de la noche.

- Fregoso, G. \&Aguilar, L.E. (2013). Algunos indicios de analfabetismo funcional en México. Alabe 7. (www.revistaalabe,com)

- Goldmann, L. (1975). Marxismo y ciencias humanas. Buenos Aires: Amorrortu.

- Instituto Nacional para la Evaluación de la Educación (2OII). Panorama Educativo de México 2010. Obtenido el 3 de octubre de 2012 desde www.inee.edu.mx/index.php/publicaciones/.../panorama-educativo

- Martínez, N. (2005. I8 de septiembre). Bibliotecas públicas en el abandono. El Universal. p. II.

- Munguía, I. y otros. (20I5). Habilidades lingüísticas de los estudiantes de primer ingreso a las instituciones de educación superior. México: Asociación Nacional de Universidades e Instituciones de Enseñanza Superior.

- Secretaría de Educación Pública. (2OII). Enlace 2006-20II: Porcentaje de alumnos por niveles de logro para cada modalidad y entidad. Primaria y secundaria. Español. México: 